\title{
Topology-Mediated Enhanced Polaron Coherence in Covalent Organic Frameworks
}

\author{
Raja Ghosh*,† and Francesco Paesani*,+, ${ }^{*}, \S$ \\ $\dagger$ Department of Chemistry and Biochemistry, University of California San Diego, \\ La Jolla, California 92093, United States \\ $\ddagger$ Department of Chemistry and Biochemistry, University of California, San Diego \\ La Jolla, California 92093, United States \\ \Materials Science and Engineering, University of California, San Diego \\ La Jolla, California 92093, United States \\ $\S$ San Diego Supercomputer Center, University of California, San Diego \\ La Jolla, California 92093, United States \\ E-mail: raghosh@ucsd.edu; fpaesani@ucsd.edu
}




\begin{abstract}
We employ the Holstein model for polarons to investigate the relationship among defects, topology, Coulomb trapping, and polaron delocalization in covalent organic frameworks (COFs). We find that intra-sheet topological connectivity and $\pi$-column density can override disorder-induced deep traps and significantly enhance polaron migration by several orders of magnitude in good agreement with recent experimental observations. The combination of percolation networks and micropores makes trigonal COFs ideally suited for charge transport followed by kagome/tetragonal, and hexagonal structures. By comparing the polaron spectral signatures and coherence numbers of large 3D frameworks having a maximum of 180 coupled chromophores, we show that controlling nanoscale defects and the location of the counter anion is critical for the design of new COF-based materials yielding higher mobilities. Our analysis establishes design strategies for enhanced conductivity in COFs which can be readily generalized to other classes of conductive materials such as metal-organic frameworks and perovskites.
\end{abstract}

\title{
Graphical TOC Entry
}

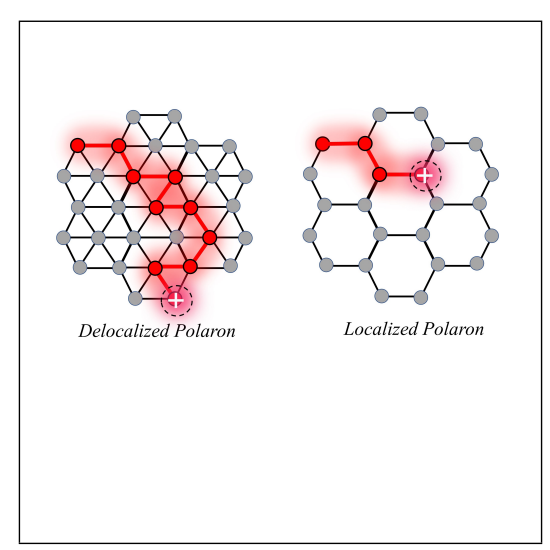


Since their inception in 2016, ${ }^{1,2} \mathrm{sp}^{2}$-carbon conjugated covalent organic frameworks (COFs) have witnessed a rapid surge in research interest, mainly driven by potential applications of these materials in optoelectronics, spintronics, chemical sensors, solar cells, and energy storage. ${ }^{3-14}$ Most of these applications require a fundamental understanding of the underlying charge transport physics and structure-property-composition relationship for the design and processing of new materials. Due to their high crystallinity, two-dimensional (2D) $\pi$ electronic communication, and topological networks, $\mathrm{sp}^{2}$ carbon-conjugated COFs provide more efficient migration of both electrons and holes than COFs based on other linkages. ${ }^{15}$ Unraveling how the complex interplay between electron-phonon coupling, defects, topology, and Coulomb trapping impacts nanoscale coherence and bulk mobility/conductivity remains elusive. ${ }^{16}$ Topology-dependent exciton and hole migration was recently investigated thanks to the designed synthesis of $\mathrm{sp}^{2}$ carbon-conjugated COFs with different topologies which were found to display high hole mobility. ${ }^{5,9}$ Despite the specific topological connectivity in a given COF has been shown, experimentally, to play a key role in determining the intrinsic charge transport properties of the framework, the number of theoretical studies aimed at characterizing the effects of the conjugated networks on polaron delocalization and photophysics of COFs is limited.

The infrared absorption spectrum of polarons in 2D COFs displays two distinct peaks, A and B. ${ }^{17,18}$ Theoretically, the red shift of peak B and the increase in the A/B peak ratio was associated with enhanced polaron coherence. ${ }^{17}$ Upon chemical doping with iodine, it was found that the absorption spectrum of TANG-COF, a (aza)triangulene-based COF, displays a blue-shifted peak B (at $\sim 1 \mathrm{eV}$ ), and a low-energy peak A centered at $\sim 0.15 \mathrm{eV}$. ${ }^{18}$ The absorption spectrum of TANG-COF was also investigated theoretically using a Holstein model based on vibrationally dressed polarons on a three-dimensional (3D) tetragonal COF lattice. ${ }^{17}$ The simulated spectrum was found to accurately reproduce the experimental positions and relative intensities of both peaks A and B. Importantly, the theoretical model was able to explain the origin of both peaks, demonstrating that the peaks A and B arise from the 
inter-framework and intra-framework components of the simulated spectrum, respectively.

In this study, we analyze the properties of "hole" polarons, which give rise to the two distinct spectral signatures in the infrared absorption spectra of 2D COFs, and elucidate the associated charge transport mechanisms. To this end, we extend the Holstein model represented in a multiparticle basis set, ${ }^{17,19}$ to enable the description of 2D COF structures with different topologies. Our model correctly represents the most important physical processes describing polarons, such as electronic coupling, vibronic coupling, and defects on equal footing, without invoking the Born-Oppenheimer approximation. ${ }^{17,20-25}$ More importantly, our model provides direct correlations between spectral signatures and nanoscale coherence as detailed in the Supporting Information. Knowing the coherence distribution in COFs is key to identifying the factors that limit charge transport in these materials - be them defects, Coulomb binding, or topological connectivity - which is the primary goal of this study.

Figure 1b shows the simulated infrared absorption spectrum for a hole in a single-layered defect-free tetragonal COF structure. In the absence of defects, the absorption spectrum responsible for the delocalized polaron is unstable and strongly dependent on the size of the crystalline domain. The simulated spectrum in Figure 1b continues to redshift and grow in intensity with increasing domain size and expanding polaron coherence. As demonstrated both experimentally ${ }^{26-28}$ and theoretically, ${ }^{21,22,29}$ charge transport in organic materials is determined by the localization of electronic states induced by lattice disorder and is not limited by electron-phonon coupling. Hence, for a defect-free COF layer, the role played by a specific connectivity becomes irrelevant since the hole can readily take any available pathway and delocalize over the entire framework. The situation is quite different, and more realistic, in the presence of short-range site disorder, since, in this case, the hole coherence is effectively determined by the degree of disorder. Intrinsic energetic disorder in organic materials arises from random fluctuations in the hole energies and can be modeled efficiently by considering a Gaussian distribution of site energies having standard deviation $\sigma$ as discussed in the Supporting Information. ${ }^{19,30-33}$ Polaron coherence stabilizes with increasing site disorder as 

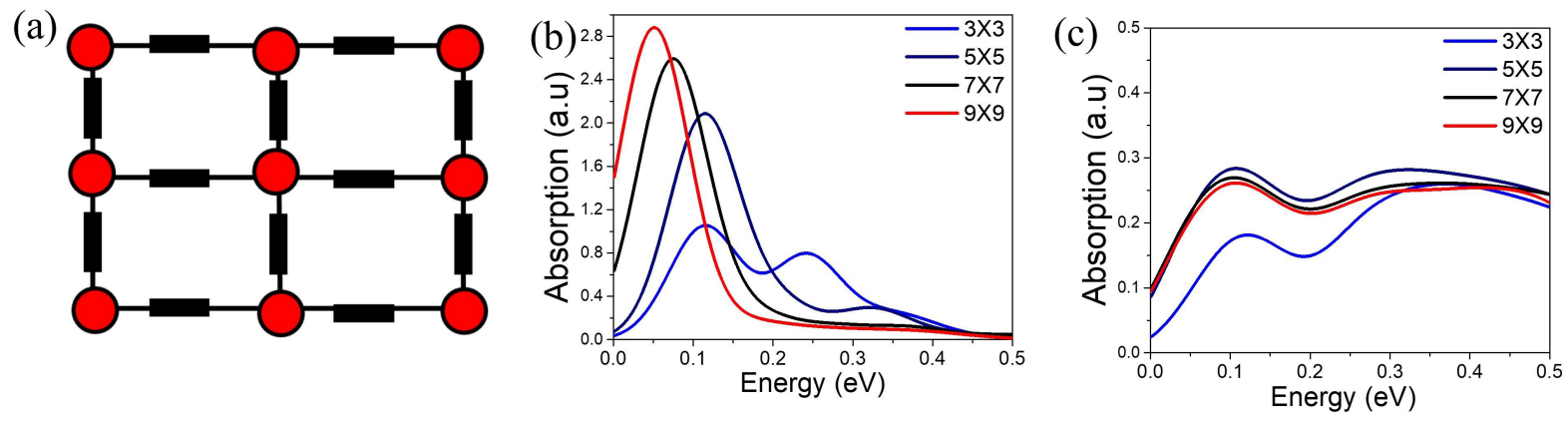

Figure 1: (a) A model $5 \times 5$ COF layer. (b) Simulated hole absorption spectrum for COF layers of various sizes (Figure S7) with no disorder $(\sigma=0 \mathrm{eV})$ and (c) with $\sigma=0.3 \mathrm{eV}$. All parameters defining the Hamiltonians used in these calculations are given in the Supporting Information.

the holes get trapped and the spectrum starts to converge. For a disorder width $(\sigma)$ of 0.3 $\mathrm{eV}$ in a tetragonal lattice, the spectrum is converged for a domain size having 21 coupled units such that the total polaron wavefunction/cloud is approximately spread over 6.7 units along the COF layer(Figure 1c).

We begin our analysis by comparing the simulated (unpolarized) infrared absorption spectrum and corresponding coherence lengths for a "free" hole (i.e., unbound to an anion) in a disordered single-layered COF having tetragonal, kagome, and trigonal topologies. We use domain sizes large enough to obtain spectral convergence and solely focus on the effect of intra-sheet connectivity on the extent of polaron coherence. Since the nearest neighbours (n.n.) across all topologies are equidistant and set to unity, we can use the same values of n.n. electronic couplings calculated for prototypical COF structures in Ref. 34. We set $\sigma=0.3 \mathrm{eV}$, since in our previous studies we found that the best agreement with the experimental spectra was obtained using $0.25 \mathrm{eV} \leq \sigma \leq 0.35 \mathrm{eV} \cdot{ }^{17,21,22}$ As shown in Figure 2a, the absorption spectrum calculated for a trigonal topology converges to a red-shifted peak $\mathrm{B}(0.26 \mathrm{eV})$ and a greater A/B peak ratio compared to the corresponding spectra calculated for tetragonal $(0.31$ $\mathrm{eV})$ and hexagonal $(0.55 \mathrm{eV})$ topologies. A trigonal framework facilitates enhanced polaron delocalization by taking advantage of better in-plane connectivity and, consequently, requires a larger domain size for the spectrum to converge compared to the other two lattice topologies 

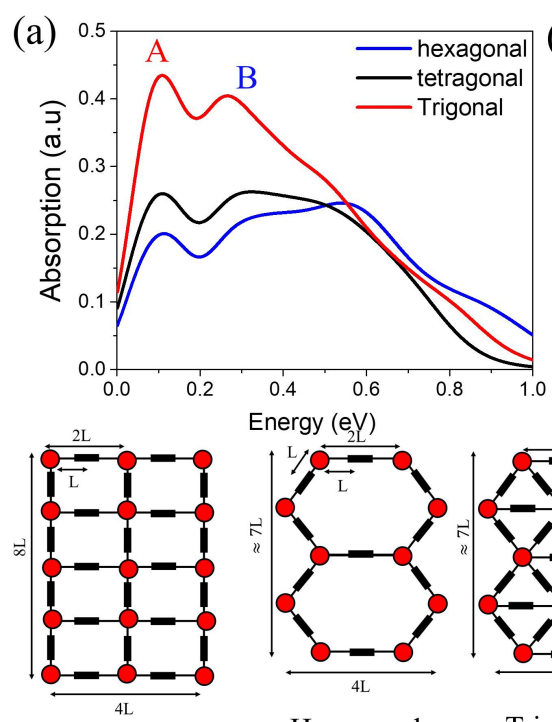

Tetragonal

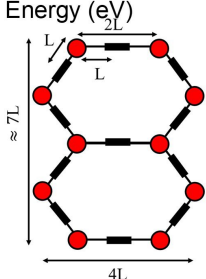

Hexagonal (b)
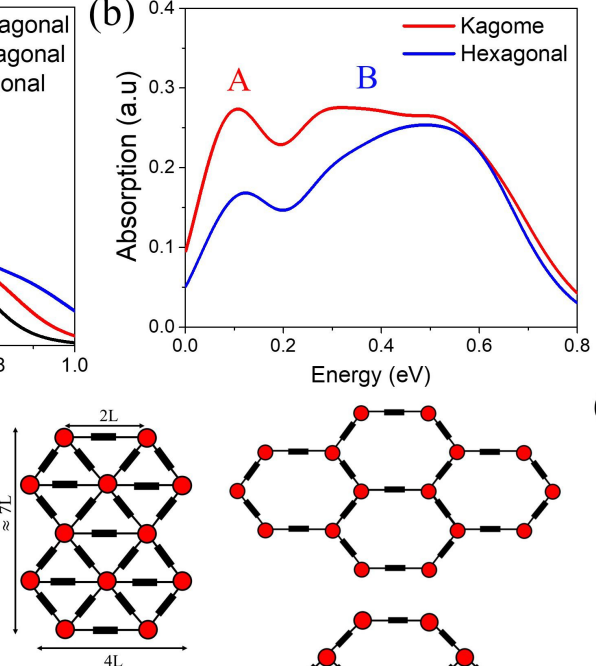

Trigonal

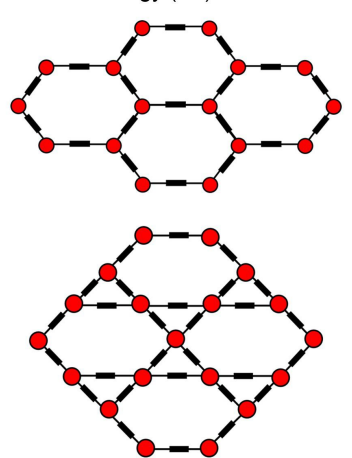

Kagome (c)

Configuration 1

(d)
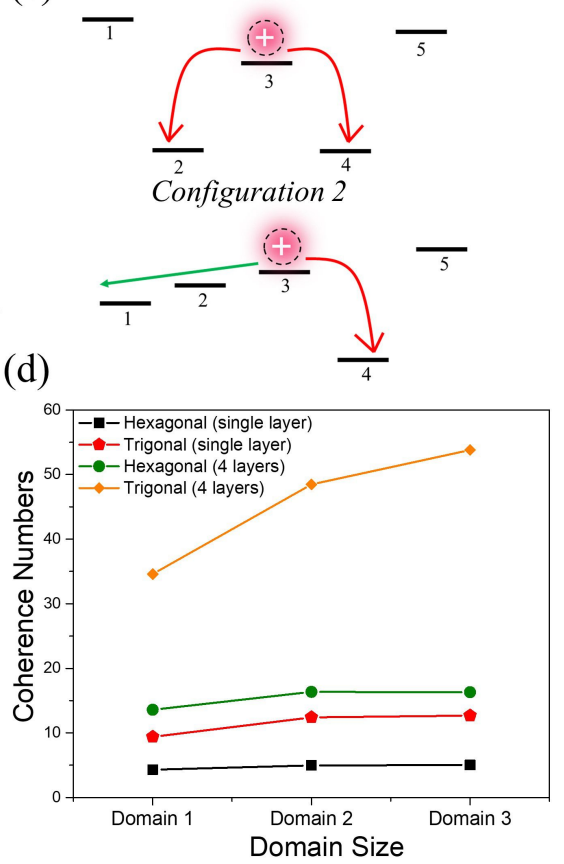

Figure 2: (a, b) Simulated hole absorption spectrum for various COF topologies. (c) Representation of site energy fluctuations due to static site disorder. (d) Calculated coherence numbers for hexagonal and trigonal topologies for domains shown in Figure S1.

(Figure S1). The effect of connectivity can be readily appreciated by comparing the schematic disorder configurations in Fig. 2c. In contrast to configuration 1, where the hole on site 3 gets trapped on moving one unit on either side, configuration 2 displays one energetically accessible pathway for the hole to delocalize, which thus prevents the hole from becoming trapped. Similarly, a hole in a trigonal lattice having six connecting pathways has a much higher probability to find a neighboring site with a favorable electronic site energy compared to a hole on a hexagonal lattice which has only three available pathways at any given point of time and hence can delocalize farther. In order to further illustrate this hypothesis, in Figure 2b, we compare the spectral signatures and the coherence lengths of hexagonal and dual-pore kagome structures calculated for large domains. While smaller domains do not have much of an advantage (Figure S2), extended kagome structures, which display four connecting pathways, enable larger polaron coherence and display a red-shifted peak B compared to hexagonal lattices. This analysis thus shows that topological connectivity helps 
circumvent trap sites and enhances polaron migration by providing alternate "defect-guided" percolation pathways.

2D-polymers can efficiently combine in-plane and out-of-plane $\pi$-electronic communication. As demonstrated schematically in Figures S3, switching the electronic coupling on along the COF layer results in an increase in columnar hole movement. In this context, trigonal topologies have an additional advantage due to the presence of higher $\pi$-column density compared to other topologies. In Figure 2d, we compare the coherence lengths for singleand multi-layered trigonal and hexagonal topologies as a function of increasing domain size. The trend in the oscillator strength is also reflected in the corresponding coherence numbers, with the intra-framework coherence calculated for the trigonal topology converging to a value that is 2.5 times larger than the value calculated for the hexagonal topology. We find that the total polaron coherence in a trigonal COF having four stacked layers is $\approx$ 3.5-4 times larger than for a hexagonal COF with four layers (Figure 2d). In our analysis, the coherence numbers are compared for single crystalline domains. However, in real COF samples, the presence of multiple isolated domains can result in a substantial increase in hole mobility. Our predicted enhancement is indeed plausible, since the HBC-COF, which has a trigonal topology, exhibits significantly higher hole mobility compared to other structures. ${ }^{9}$ We emphasize that the higher hole mobility observed in trigonal HBC-COF is not a straightforward consequence of enhanced $\pi$-electronic communication, rather it results from the interplay between electronic coupling and the presence of intrinsic defects. The hole mobility is certainly dependent on the magnitude of coupling between n.n; however, topology optimization alone can enhance hole delocalization. Our results suggest that research efforts should therefore be directed at increasing the number of electronically coupled pathways, e.g., by bridge functionalization, ${ }^{35-38}$ cross linking polymers, ${ }^{39}$ selection of superatoms, ${ }^{40}$ or exploring new molecular knots. ${ }^{9}$ It should be noted that, a recent investigation of topologydependent exciton migration found similar trends in spectral signatures as those obtained here using our theoretical model. ${ }^{5}$ However, exciton absorption for COF aggregates can be 
complicated since the nature of stacking (H vs. J aggregates) alone determines spectral shifts. ${ }^{41,42}$ The effects of $\mathrm{H}$ - and J-type stacking will be the subject of a future study. There are, however, no H- or J-type polarons. ${ }^{19}$

Next, we investigate how the presence of the dopant counter-anion impacts polaron delocalization. Introduction of the counter anion induces additional localization besides that due to the presence of defects. Interestingly, as demonstrated below, the competition between these two localization mechanisms determines the extent of polaron coherence and the factors affecting charge transport. While iodine doping was shown to result in a large increase in conductivity, ${ }^{18,43}$ the values reported so far for known COFs are still low for most practical device applications. In order to better understand the cause behind the low conductivity, we simplify the problem by first considering a linear chain hosting a single hole with a pointcharge anion placed alongside the chain at a distance $d_{\text {anion }}$ from the chain center (Figure S5). At large $d_{\text {anion }}$ values $(>2.0 \mathrm{~nm}$ ), the polaron coherence length is solely determined by the localizing effects of disorder since, in this case, the effect of Coulomb binding is negligible. However, the electrostatic interactions start to dominate with decreasing $d_{\text {anion }}$ values and finally override disorder-induced effects at reduced hole-anion separation $\left(d_{\text {anion }}<0.4 \mathrm{~nm}\right)$.

In our subsequent analysis, we consider two cases. In the first case, we keep the chain length fixed and investigate the coherence lengths as a function of increasing disorder width $(\sigma)$. Positioning the anion at a distance of $0.35 \mathrm{~nm}$ from the midpoint of a disorder-free chain containing nine repeat units, results in the formation of a deep Coulomb well (Figure S5a). Strong Coulomb binding modifies the energy landscape by isolating the unit closest to it. The formation of a deep Coulomb well completely compensates the effect of disorder, i.e., increasing the disorder width has negligible impact on the coherence lengths. This is reflected in the coherence number which remains constant at $\approx 1.5$ units with increasing $\sigma$. Figure S5c, however, shows that larger disorder widths can broaden the density of states and allow sites with sufficiently low energy to act as bridges for the hole to jump from one Coulomb well to another. In the second case, we investigate the variation of the coherence 
(a)
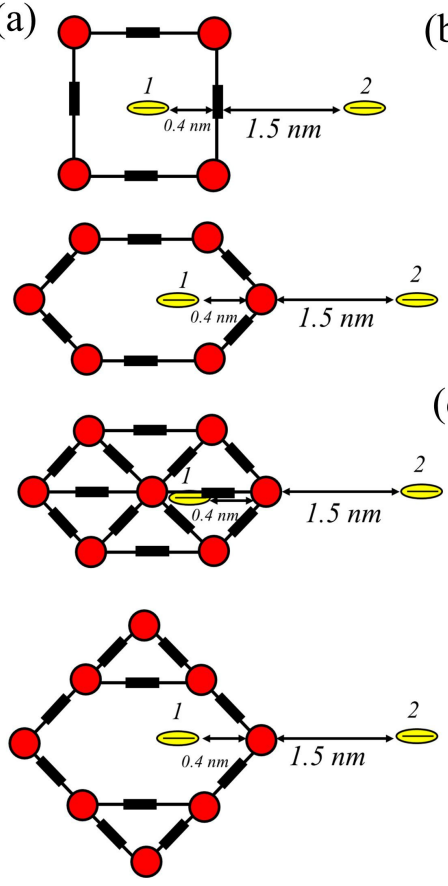

(b)

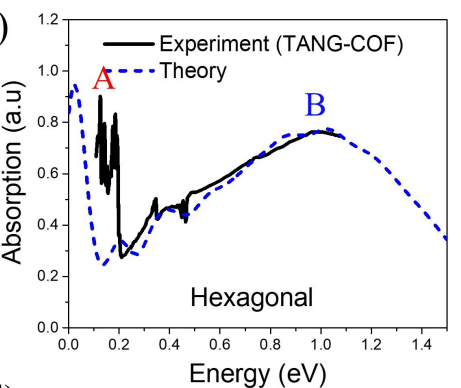

(d)

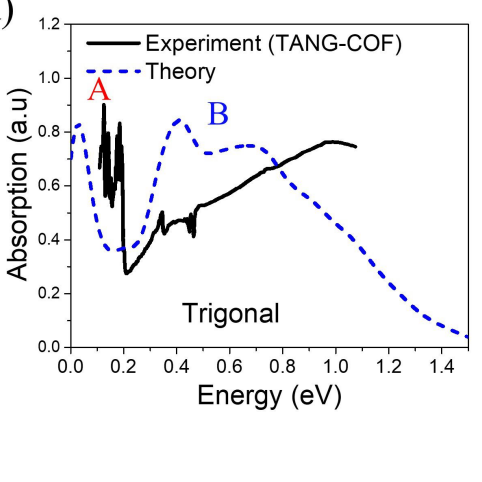

(c)

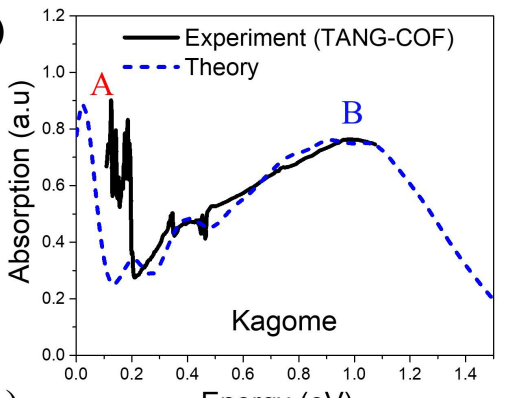

(e)

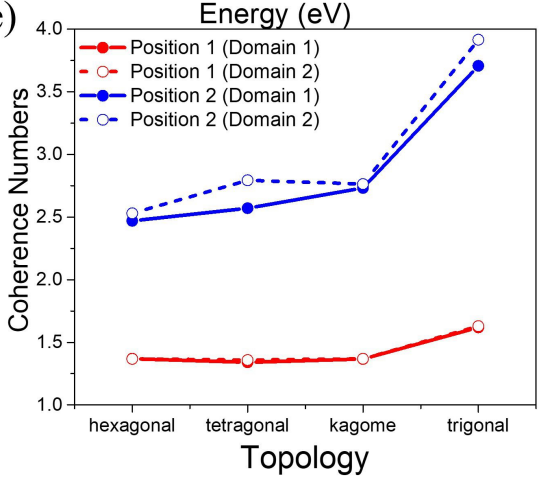

Figure 3: (a) Schematic illustration showing the two counter anion positions relative to the framework considered in our analyses. (b, c, d) Comparisons between simulated (dashed) and experimental (solid) IR spectra for iodine-doped TANG-COF. (e) Calculated coherence numbers for the two anion positions as a function of topology and domain sizes shown in Figure S8.

length as a function of increasing chain length. By setting $\sigma=0$ and keeping $d_{\text {anion }}$ fixed from the chain midpoint, we find that increasing the chain length does not have any effect on the polaron coherence numbers due to the formation of a electrostatically localized charge carrier in the Coulomb well. These model systems are quite general and can be readily extended to 2D COF structures hosting "through-bond" (intra-framework) and "throughspace" (intra-framework) electronic interactions.

In a previous study, we were able to reproduce the measured spectrum of iodine doped TANG-COF by positioning the counter anion inside the pore of an oxidized tetragonal COF lattice, at a distance of $0.35-0.45 \mathrm{~nm}$ from the framework backbone ${ }^{17}$ Since the 2D version of TANG-COF results in a hexagonal arrangement, ${ }^{18}$ we report here simulations for this structure as well as other topologies. Additional insights into the structure-property relationship along with all simulation details are presented in the Supporting Information. 
Figures $3 \mathrm{~b}, \mathrm{c}$ show that the agreement with the experimental data is only marginally improved when employing hexagonal and kagome topologies compared to the results presented in Ref. 17 for the tetragonal topology. However, using identical parameters, the trigonal topology displays a red-shifted peak B (Figure 3d). By changing the location of the counter-anion relative to the COF backbone and slightly modifying the eletronic coupling parameters, Figure S6d shows that it is possible to achieve better agreement with experiment. However, TANG-COF does not have a trigonal arrangement and, although our theoretical results for this topology are quantitatively different from those obtained for the other topologies, the overall conclusions remain unchanged.

More relevant for this study, in Figure 3e, we compare the coherence lengths calculated as a function of topology and domain size for the two counter anion positions shown in Figure 3a (Figure S8). Due to strong Coulomb binding across all topologies, the coherence lengths barely change when the counter anion is placed inside the pore in close proximity to the framework, which nullifies the effect of intra-sheet connectivity. Furthermore, Figure 3e also shows that the presence of larger domains does not provide any specific advantage as the coherence lengths remain unchanged. The effect of topology becomes more prominent in the case of moderate to weak Coulomb binding, where the polarons can take advantage of the 3D topological connectivity. This is demonstrated in Figure 3e where the coherence lengths not only steadily increase as a function of topology but also display a larger degree of delocalization with increasing domain sizes, which in turn is dependent on the magnitude of n.n. transfer integrals. In the presence of dopants, a single-layered trigonal COF displays $\approx 2.4$-fold increase in coherence relative to the hexagonal topology. We believe that these results are particularly important in the context of recent experimental efforts aimed at synthesizing COFs with a high degree of crystallinity ${ }^{44-50}$ or larger domains. ${ }^{51,52}$ While such efforts are certainly important for achieving higher hole mobility, our analyses demonstrate that, independently of the degree of crystallinity and/or domain sizes, if the counter anions residing inside the COF pores trap the free charge carriers, the conductivity of COFs will 
always be lower ${ }^{18,43,53-58}$ than that measured for doped conjugated polymers. ${ }^{25,59,60}$ Our results thus suggest that it would be important to explore the potential use of other dopants and doping techniques in order to achieve higher conductivities in COF films. ${ }^{25,61,62}$

To summarize, we have provided fundamental insights into the charge transport mechanisms in COFs. Our analyses demonstrate that the intrinsic hole mobility in COFs can be significantly enhanced by engineering topological connectivity and nanoscale defects. Our results for doped COF films indicate that controlling the precise location of the counter anion relative to the framework is crucial for enhancing the conductivity of these materials, playing a more important role than achieving higher degrees of crystallinity. We believe that our findings provide important design principles for the development of novel optoelectronic COF-based devices, such as topology-optimized solar cells and light-emitting diodes.

\section{Acknowledgement}

This research was supported by the National Science Foundation through grant no. 1704063. This research used resources of the Extreme Science and Engineering Discovery Environment (XSEDE), which is supported by the National Science Foundation grant no. ACI-1548562, the National Energy Research Scientific Computing Center (NERSC), which is supported by the Office of Science of the U.S. Department of Energy under Contract DE-AC02-05CH11231, and the Triton Shared Computing Cluster (TSCC) at the San Diego Supercomputer Center (SDSC). The software PETSc and SLEPc was used to diagonalize the Holstein Hamiltonians. ${ }^{63-65}$

\section{Supporting Information Available}

Details of the Holstein Hamiltonian, infrared absorption, coherence function, and additional comparisons with experimental data. 


\section{References}

(1) Jin, E.; Jiang, D. An All sp²-Carbon-Based Covalent Organic Framework. Proceedings of The 96th Annual Meeting of Chemical Society of Japan, 1D1-09. 2016.

(2) Zhuang, X.; Zhao, W.; Zhang, F.; Cao, Y.; Liu, F.; Bi, S.; Feng, X. A TwoDimensional Conjugated Polymer Framework with Fully sp²-Bonded Carbon Skeleton. Polym. Chem. 2016, 7, 4176-4181.

(3) Jin, E.; Asada, M.; Xu, Q.; Dalapati, S.; Addicoat, M. A.; Brady, M. A.; Xu, H.; Nakamura, T.; Heine, T.; Chen, Q. et al. Two-Dimensional sp² Carbon-Conjugated Covalent Organic Frameworks. Science 2017, 357, 673-676.

(4) Jin, E.; Li, J.; Geng, K.; Jiang, Q.; Xu, H.; Xu, Q.; Jiang, D. Designed Synthesis of Stable Light-Emitting Two-Dimensional sp² Carbon-Conjugated Covalent Organic Frameworks. Nat. Commun. 2018, 9, 1-10.

(5) Jin, E.; Geng, K.; Lee, K. H.; Jiang, W.; Li, J.; Jiang, Q.; Irle, S.; Jiang, D. Topology-Templated Synthesis of Crystalline Porous Covalent Organic Frameworks. Angew. Chem. Int. Ed. 2020, 132, 12260-12267.

(6) Xu, S.; Wang, G.; Biswal, B. P.; Addicoat, M.; Paasch, S.; Sheng, W.; Zhuang, X.; Brunner, E.; Heine, T.; Berger, R. et al. A Nitrogen-Rich 2D sp²-Carbon-Linked Conjugated Polymer Framework as a High-Performance Cathode for Lithium-Ion Batteries. Angew. Chem. Int. Ed. 2019, 131, 859-863.

(7) Chen, R.; Shi, J.-L.; Ma, Y.; Lin, G.; Lang, X.; Wang, C. Designed Synthesis of a 2D Porphyrin-Based $\mathrm{sp}^{2}$ Carbon-Conjugated Covalent Organic Framework for Heterogeneous Photocatalysis. Angew. Chem. Int. Ed. 2019, 58, 6430-6434.

(8) Cui, W.-R.; Zhang, C.-R.; Jiang, W.; Li, F.-F.; Liang, R.-P.; Liu, J.; Qiu, J.-D. Regen- 
erable and Stable $\mathrm{sp}^{2}$ Carbon-Conjugated Covalent Organic Frameworks for Selective Detection and Extraction of Uranium. Nat. Commun. 2020, 11, 1-10.

(9) Dalapati, S.; Addicoat, M.; Jin, S.; Sakurai, T.; Gao, J.; Xu, H.; Irle, S.; Seki, S.; Jiang, D. Rational Design of Crystalline Supermicroporous Covalent Organic Frameworks with Triangular Topologies. Nat. Commun. 2015, 6, 1-8.

(10) Souto, M.; Perepichka, D. F. Electrically Conductive Covalent Organic Frameworks: Bridging the Fields of Organic Metals and 2D Materials. J. Mater. Chem. C 2021,

(11) Li, S.; Li, L.; Li, Y.; Dai, L.; Liu, C.; Liu, Y.; Li, J.; Lv, J.; Li, P.; Wang, B. Fully Conjugated Donor-Acceptor Covalent Organic Frameworks for Photocatalytic Oxidative Amine Coupling and Thioamide Cyclization. ACS Catal. 2020, 10, 8717-8726.

(12) Zhao, Y.; Liu, H.; Wu, C.; Zhang, Z.; Pan, Q.; Hu, F.; Wang, R.; Li, P.; Huang, X.; Li, Z. Fully Conjugated Two-Dimensional sp2-Carbon Covalent Organic Frameworks as Artificial Photosystem I with High Efficiency. Angew. Chem. Int. Ed. 2019, 131, $5430-5435$.

(13) Jin, E.; Lan, Z.; Jiang, Q.; Geng, K.; Li, G.; Wang, X.; Jiang, D. 2D sp2 CarbonConjugated Covalent Organic Frameworks for Photocatalytic Hydrogen Production from Water. Chem 2019, 5, 1632-1647.

(14) Xia, C.; Kirlikovali, K. O.; Nguyen, T. H. C.; Nguyen, X. C.; Tran, Q. B.; Duong, M. K.; Dinh, M. T. N.; Nguyen, D. L. T.; Singh, P.; Raizada, P. et al. The Emerging Covalent Organic Frameworks (COFs) for Solar-Driven Fuels Production. Coord. Chem. Rev. 2021, 446, 214117.

(15) He, T.; Geng, K.; Jiang, D. All sp² Carbon Covalent Organic Frameworks. Trends Chem. 2021, 
(16) Hendon, C. H.; Rieth, A. J.; Korzyński, M. D.; Dincă, M. Grand Challenges and Future Opportunities for Metal-Organic Frameworks. ACS Cent. Sci. 2017, 3, 554-563.

(17) Ghosh, R.; Paesani, F. Unraveling the Effect of Defects, Domain Size, and Chemical Doping on Photophysics and Charge Transport in Covalent Organic Frameworks. Chem. Sci. 2021, 12, 8373-8384.

(18) Lakshmi, V.; Liu, C.-H.; Rajeswara Rao, M.; Chen, Y.; Fang, Y.; Dadvand, A.; Hamzehpoor, E.; Sakai-Otsuka, Y.; Stein, R. S.; Perepichka, D. F. A Two-Dimensional Poly(azatriangulene) Covalent Organic Framework with Semiconducting and Paramagnetic States. J. Am. Chem. Soc. 2020, 142, 2155-2160.

(19) Ghosh, R.; Spano, F. C. Excitons and Polarons in Organic Materials. Acc. Chem. Res. 2020, 53, 2201-2211.

(20) Ghosh, R.; Pochas, C. M.; Spano, F. C. Polaron Delocalization in Conjugated Polymer Films. J. Phys. Chem. C 2016, 120, 11394-11406.

(21) Ghosh, R.; Chew, A. R.; Onorato, J.; Pakhnyuk, V.; Luscombe, C. K.; Salleo, A.; Spano, F. C. Spectral Signatures and Spatial Coherence of Bound and Unbound Polarons in P3HT Films: Theory Versus Experiment. J. Phys. Chem. C 2018, 122, $18048-18060$.

(22) Ghosh, R.; Luscombe, C. K.; Hambsch, M.; Mannsfeld, S. C.; Salleo, A.; Spano, F. C. Anisotropic Polaron Delocalization in Conjugated Homopolymers and Donor-Acceptor Copolymers. Chem. Mater. 2019, 31, 7033-7045.

(23) Chew, A. R.; Ghosh, R.; Shang, Z.; Spano, F. C.; Salleo, A. Sequential Doping Reveals the Importance of Amorphous Chain Rigidity in Charge Transport of Semi-Crystalline Polymers. J. Phys. Chem. Lett. 2017, 8, 4974-4980. 
(24) Chew, A. R.; Ghosh, R.; Pakhnyuk, V.; Onorato, J.; Davidson, E. C.; Segalman, R. A.; Luscombe, C. K.; Spano, F. C.; Salleo, A. Unraveling the Effect of Conformational and Electronic Disorder in the Charge Transport Processes of Semiconducting Polymers. Adv. Funct. Mater. 2018, 28, 1804142.

(25) Aubry, T. J.; Axtell, J. C.; Basile, V. M.; Winchell, K.; Lindemuth, J. R.; Porter, T. M.; Liu, J.-Y.; Alexandrova, A. N.; Kubiak, C. P.; Tolbert, S. H. et al. Dodecaborane-Based Dopants Designed to Shield Anion Electrostatics Lead to Increased Carrier Mobility in a Doped Conjugated Polymer. Adv. Mater. 2019, 31, 1805647.

(26) Noriega, R.; Rivnay, J.; Vandewal, K.; Koch, F. P.; Stingelin, N.; Smith, P.; Toney, M. F.; Salleo, A. A General Relationship Between Disorder, Aggregation and Charge Transport in Conjugated Polymers. Nat. Mater. 2013, 12, 1038-1044.

(27) Sirringhaus, H. Device Physics of Solution-Processed Organic Field-Effect Transistors. Adv. Mater. 2005, 17, 2411-2425.

(28) Chang, J.-F.; Sirringhaus, H.; Giles, M.; Heeney, M.; McCulloch, I. Relative Importance of Polaron Activation and Disorder on Charge Transport in High-Mobility Conjugated Polymer Field-Effect Transistors. Phys. Rev. B 2007, 76, 205204.

(29) Fornari, R. P.; Troisi, A. Narrower Bands with Better Charge Transport: The Counterintuitive Behavior of Semiconducting Copolymers. Adv. Mater. 2014, 26, 7627-7631.

(30) Shen, Y.; Diest, K.; Wong, M. H.; Hsieh, B. R.; Dunlap, D. H.; Malliaras, G. G. Charge Transport in Doped Organic Semiconductors. Phys. Rev. B 2003, 68, 081204.

(31) Schwarze, M.; Gaul, C.; Scholz, R.; Bussolotti, F.; Hofacker, A.; Schellhammer, K. S.; Nell, B.; Naab, B. D.; Bao, Z.; Spoltore, D. et al. Molecular Parameters Responsible for Thermally Activated Transport in Doped Organic Semiconductors. Nat. Mater. 2019, 18, 242-248. 
(32) Bässler, H. Localized States and Electronic Transport in Single Component Organic Solids with Diagonal Disorder. Phys. Status Solidi B 1981, 107, 9-54.

(33) Hoffmann, S. T.; Bässler, H.; Köhler, A. What Determines Inhomogeneous Broadening of Electronic Transitions in Conjugated Polymers? J. Phys. Chem. B 2010, 114, 1703717048.

(34) Thomas, S.; Li, H.; Dasari, R. R.; Evans, A. M.; Castano, I.; Allen, T. G.; Reid, O. G.; Rumbles, G.; Dichtel, W. R.; Gianneschi, N. C. et al. Design and Synthesis of TwoDimensional Covalent Organic Frameworks with Four-Arm Cores: Prediction of Remarkable Ambipolar Charge-Transport Properties. Mater. Horiz. 2019, 6, 1868-1876.

(35) Keller, N.; Bessinger, D.; Reuter, S.; Calik, M.; Ascherl, L.; Hanusch, F. C.; Auras, F.; Bein, T. Oligothiophene-bridged Conjugated Cvalent Organic Frameworks. J. Am. Chem. Soc. 2017, 139, 8194-8199.

(36) Li, K.; Wong, N. K.; Strauss, M. J.; Evans, A. M.; Matsumoto, M.; Dichtel, W. R.; Adronov, A. Postsynthetic Modification of a Covalent Organic Framework Achieved via Strain-Promoted Cycloaddition. J. Am. Chem. Soc. 2021, 143, 649-656.

(37) Gatou, M.-A.; Bika, P.; Stergiopoulos, T.; Dallas, P.; Pavlatou, E. A. Recent Advances in Covalent Organic Frameworks for Heavy Metal Removal Applications. Energies 2021, 14, 3197 .

(38) Kuc, A.; Springer, M. A.; Batra, K.; Juarez-Mosqueda, R.; Wöll, C.; Heine, T. Proximity Effect in Crystalline Framework Materials: Stacking-Induced Functionality in MOFs and COFs. Adv. Funct. Mater. 2020, 30, 1908004.

(39) Jadhav, T.; Fang, Y.; Liu, C.-H.; Dadvand, A.; Hamzehpoor, E.; Patterson, W.; Jonderian, A.; Stein, R. S.; Perepichka, D. F. Transformation Between 2D and 3D Covalent Organic Frameworks via Reversible [2+2] Cycloaddition. J. Am. Chem. Soc. 2020, 142, 8862-8870. 
(40) Jing, Y.; Heine, T. Two-Dimensional Kagome Lattices Made of Hetero Triangulenes Are Dirac Semimetals or Single-Band Semiconductors. J. Am. Chem. Soc. 2018, 141, $743-747$.

(41) Spano, F. C. The Spectral Signatures of Frenkel Polarons in H- and J-Aggregates. Acc. Chem. Res. 2010, 43, 429-439.

(42) Hestand, N. J.; Spano, F. C. Expanded Theory of H- and J-Molecular Aggregates: The Effects of Vibronic Coupling and Intermolecular Charge Transfer. Chem. Rev. 2018, 118, 7069-7163.

(43) Wang, M.; Wang, M.; Lin, H.-H.; Ballabio, M.; Zhong, H.; Bonn, M.; Zhou, S.; Heine, T.; Cánovas, E.; Dong, R. et al. High-mobility semiconducting two-dimensional conjugated covalent organic frameworks with p-type doping. J. Am. Chem. Soc. 2020, 142, 21622-21627.

(44) Li, X.; Qiao, J.; Chee, S. W.; Xu, H.-S.; Zhao, X.; Choi, H. S.; Yu, W.; Quek, S. Y.; Mirsaidov, U.; Loh, K. P. Rapid, Scalable Construction of Highly Crystalline Acylhydrazone Two-dimensional Covalent Organic Frameworks via Dipole-Induced Antiparallel Stacking. J. Am. Chem. Soc. 2020, 142, 4932-4943.

(45) Xu, L.; Ding, S.-Y.; Liu, J.; Sun, J.; Wang, W.; Zheng, Q.-Y. Highly Crystalline Covalent Organic Frameworks from Flexible Building Blocks. Chem. Commun. 2016, 52, 4706-4709.

(46) Uribe-Romo, F. J.; Hunt, J. R.; Furukawa, H.; Klock, C.; O'Keeffe, M.; Yaghi, O. M. A Crystalline Imine-Linked 3-D Porous Covalent Organic Framework. J. Am. Chem. Soc. 2009, 131, 4570-4571.

(47) Uribe-Romo, F. J.; Doonan, C. J.; Furukawa, H.; Oisaki, K.; Yaghi, O. M. Crystalline Covalent Organic Frameworks with Hydrazone Linkages. J. Am. Chem. Soc. 2011, 133, $11478-11481$. 
(48) Burke, D. W.; Sun, C.; Castano, I.; Flanders, N. C.; Evans, A. M.; Vitaku, E.; McLeod, D. C.; Lambeth, R. H.; Chen, L. X.; Gianneschi, N. C. et al. Acid Exfoliation of Imine-linked Covalent Organic Frameworks Enables Solution Processing into Crystalline Thin Films. Angew. Chem. Int. Ed. 2020, 59, 5165-5171.

(49) Li, C.; Yu, G. Controllable Synthesis and Performance Modulation of 2D CovalentOrganic Frameworks. Small 2021, 2100918.

(50) Yang, J.; Kang, F.; Wang, X.; Zhang, Q. Design Strategies of Improving the Crystallinity of Covalent Organic Frameworks and Conjugated Polymers: A Review. Mater. Horiz. 2021,

(51) Auras, F.; Ascherl, L.; Hakimioun, A. H.; Margraf, J. T.; Hanusch, F. C.; Reuter, S.; Bessinger, D.; Döblinger, M.; Hettstedt, C.; Karaghiosoff, K. et al. Synchronized Offset Stacking: A Concept for Growing Large-Domain and Highly Crystalline 2D Covalent Organic Frameworks. J. Am. Chem. Soc. 2016, 138, 16703-16710.

(52) Flanders, N. C.; Kirschner, M. S.; Kim, P.; Fauvell, T. J.; Evans, A. M.; Helweh, W.; Spencer, A. P.; Schaller, R. D.; Dichtel, W. R.; Chen, L. X. Large Exciton Diffusion Coefficients in Two-Dimensional Covalent Organic Frameworks with Different Domain Sizes Revealed by Ultrafast Exciton Dynamics. J. Am. Chem. Soc. 2020,

(53) Meng, Z.; Stolz, R. M.; Mirica, K. A. Two-Dimensional Chemiresistive Covalent Organic Framework with High Intrinsic Conductivity. J. Am. Chem. Soc. 2019, 141, 1192911937.

(54) Wang, L.; Dong, B.; Ge, R.; Jiang, F.; Xu, J. Fluorene-Based Two-Dimensional Covalent Organic Framework with Thermoelectric Properties Through Doping. ACS Appl. Mater. Interfaces 2017, 9, 7108-7114.

(55) Cai, S.-L.; Zhang, Y.-B.; Pun, A. B.; He, B.; Yang, J.; Toma, F. M.; Sharp, I. D.; Yaghi, O. M.; Fan, J.; Zheng, S.-R. et al. Tunable Electrical Conductivity in Oriented 
Thin Films of Tetrathiafulvalene-Based Covalent Organic Framework. Chem. Sci. 2014, $5,4693-4700$.

(56) Wang, C.; Wang, Y.; Ge, R.; Song, X.; Xing, X.; Jiang, Q.; Lu, H.; Hao, C.; Guo, X.; Gao, Y. et al. A 3D Covalent Organic Framework with Exceptionally High Iodine Capture Capability. Chem. Eur. J. 2018, 24, 585-589.

(57) Li, H.; Chang, J.; Li, S.; Guan, X.; Li, D.; Li, C.; Tang, L.; Xue, M.; Yan, Y.; Valtchev, V. et al. Three-Dimensional Tetrathiafulvalene-Based Covalent Organic Frameworks for Tunable Electrical Conductivity. J. Am. Chem. Soc. 2019, 141, 1332413329.

(58) Rotter, J. M.; Guntermann, R.; Auth, M.; Mähringer, A.; Sperlich, A.; Dyakonov, V.; Medina, D. D.; Bein, T. Highly Conducting Wurster-Type Twisted Covalent Organic Frameworks. Chem. Sci. 2020, 11, 12843-12853.

(59) Shirakawa, H.; Louis, E. J.; MacDiarmid, A. G.; Chiang, C. K.; Heeger, A. J. Synthesis of Electrically Conducting Organic Polymers: Halogen Derivatives of Polyacetylene, $(\mathrm{CH})_{x}$. J. Chem. Soc., Chem. Commun. 1977, 578-580.

(60) Kim, N.; Kee, S.; Lee, S. H.; Lee, B. H.; Kahng, Y. H.; Jo, Y.-R.; Kim, B.-J.; Lee, K. Highly Conductive PEDOT: PSS Nanofibrils Induced by Solution-Processed Crystallization. Adv. Mater. 2014, 26, 2268-2272.

(61) Ma, Z.; Shi, W.; Yan, K.; Pan, L.; Yu, G. Doping Engineering of Conductive Polymer Hydrogels and their Application in Advanced Sensor Technologies. Chem. Sci. 2019, 10, 6232-6244.

(62) Liu, J.; Garman, M. P.; Dong, J.; van der Zee, B.; Qiu, L.; Portale, G.; Hummelen, J. C.; Koster, L. J. A. Doping Engineering Enables Highly Conductive and Thermally Stable n-Type Organic Thermoelectrics with High Power Factor. ACS Appl. Energy Mater. 2019, 2, 6664-6671. 
(63) Hernandez, V.; Roman, J. E.; Vidal, V. SLEPc: A Scalable and Flexible Toolkit for the Solution of Eigenvalue Problems. ACM Trans. Math. Software 2005, 31, 351-362.

(64) Roman, J. E.; Campos, C.; Romero, E.; Tomas, A. SLEPc Users Manual; 2020.

(65) Balay, S.; Abhyankar, S.; Adams, M. F.; Brown, J.; Brune, P.; Buschelman, K.; Dalcin, L.; Dener, A.; Eijkhout, V.; Gropp, W. D. et al. PETSc Web page. https: //www.mcs.anl.gov/petsc, 2019; https://www.mcs .anl.gov/petsc. 